\title{
Space and Atmospheric Environments: from Low Earth Orbits to Deep Space
}

\author{
Janet L. Barth \\ NASA/Goddard Space Flight Center
}

\begin{abstract}
Natural space and atmospheric environments pose a difficult challenge for designers of technological systems in space. The deleterious effects of environment interactions with the systems include degradation of materials, thermal changes, contamination, excitation, spacecraft glow, charging, radiation damage, and induced background interference. Design accommodations must be realistic with minimum impact on performance while maintaining a balance between cost and risk. The goal of applied research in space environments and effects is to limit environmental impacts at low cost relative to spacecraft cost and to infuse enabling and commercial off-the-shelf technologies into space programs. The need to perform applied research to understand the space environment in a practical sense and to develop methods to mitigate these environment effects is frequently underestimated by space agencies and industry. Applied science research in this area is critical because the complexity of spacecraft systems is increasing, and they are exposed simultaneously to a multitude of space environments.
\end{abstract}

\section{INTRODUCTION}

Spacecraft are exposed to a multitude of environments that are not present at the surface of the Earth, including plasmas, high-energy charged particles, neutral gases, x-rays, ultraviolet (UV) irradiation, meteoroids, and orbital debris. The interaction of these environments with spacecraft systems cause degradation of materials, thermal changes, contamination, excitation, spacecraft glow, charging, radiation damage, and induced background interference. The damaging effects of natural space and atmospheric environments pose a difficult challenge for spacecraft designers. Unfortunately, the need to perform applied research to understand and model the space environments, to understand the physics of the interaction of the environment with spacecraft systems, and to develop methods to mitigate environmental effects is frequently underestimated by space agencies and industry.

At the same time that the complexity and performance requirements of spacecraft systems are increasing, other system drivers reduce our ability to meet requirements. For example, the demand for commercial microelectronics reduces the availability of components suitable for space environments. Also, the need to design lighter and more complex spacecraft structures pushes the development of exotic materials for space use. Uncertainties in space environment and effects models used to predict the performance of new technologies translate into large design margins. Large design margins can preclude the use of new technologies that will meet mission requirements. The goal of applied research in space environments and effects is to reduce design margins used to account for the uncertainty of performance predictions, thereby enabling technology infusion into space programs.

Design accommodations must have minimum impact on performance and budgets. The challenge is to achieve a realistic balance between cost of environment accommodations and mission risk. Several organizations have developed concurrent engineering approaches to accommodating environment effects and assessing risk. [1,2] Regardless of the environmental effect or the technology, the approaches follow these steps:

- Define the environment external to the spacecraft;

- Evaluate the environment interaction with the spacecraft; 
- Define the requirements and define criticality factors;

- Evaluate design and performance characteristics of components;

- "Engineer" with designers and program managers including risk analysis and definition of design margins;

- Iterate the process with updated knowledge.

Note that the first step for every mission is to define the level of the environments in metrics that are applicable the effect on the spacecraft technologies. This is accomplished by using environment and interaction models, and when models are not available, by using in-flight data. The purpose of this paper is to describe the natural space environments that must be taken into account when designing spacecraft. Specific effects on materials and structures can be found in other contributions to these proceedings. Further information on the radiation environment can be found in Reference 3 and information on radiation effects can be found in Reference 4.

\section{DESCRIPTION OF THE ENVIRONMENTS}

The space and atmospheric environments relevant to spacecraft effects can be roughly categorized into meteoroid and debris, ultraviolet irradiation, neutral thermosphere, cold and hot plasma, and particle radiation. The differences between the atmospheres and magnetospheres of the planets and interplanetary space are dramatic. Even within the atmospheric and magnetospheric systems of the Earth, there are large spatial and temporal variations in the constituency and density of the environments.

For the purpose of discussion of environmental definitions, missions can be roughly categorized into low earth orbits (LEOs), middle earth orbits (MEOs), geosynchronous (GEO), geosynchronous transfer orbits (GTOs), interplanetary, and other planets. Table 1 gives a summary of the differences between the space environments around Earth and other planets

Table 1: Variation of planetary environments from Earth

\begin{tabular}{||c|c|}
\hline ENVIRONMENT & COMPARISON TO EARTH \\
\hline Solar Wind & $\sim$ Same \\
\hline Meteoroids & $\sim$ Same \\
\hline Orbital Debris & None at this time \\
\hline Galactic Cosmic Rays & Small variation \\
\hline Solar Particles & Large variation with radial distance from Sun \\
\hline Solar Radiance & Large variation with radial distance from Sun \\
\hline Atmospheres & Large variations \\
\hline Trapped Radiation & Large variations \\
\hline
\end{tabular}

\subsection{The Solar Influence on Space Environments}

The complex environment of Sun-Earth space consists of time varying ultraviolet, $x$-ray, plasma, and high-energy particle environments. Variations depend on location in space and on the year in the solar cycle, both somewhat predictable. However, large variations that depend on events on the Sun are not predictable with reasonable certainty and are known only statistically based on past history. Because the Sun is a gas, its solar magnetic field is convoluted and highly variable. Both the long-term variation in the magnetic field that occurs in a 22-year cycle and the short term variations in the form of intense, short lived storms are responsible for observable changes in the interplanetary and near-Earth environments. 
The sun's outer atmosphere, the corona (see Figure 1), extends several solar diameters into interplanetary space. The corona continuously emits a stream of protons, electrons, doubly charged helium ions, and small amounts of other heavy ions, collectively called the solar wind. It was once thought that the region where the solar wind could no longer be detected, i.e., the boundary of the heliosphere, was not far beyond Jupiter ( 800 million $\mathrm{km}$ ). However, the Pioneer 10 spacecraft, presently at $>12$ billion kilometers from Earth, is still measuring solar wind. Scientists now believe that the boundary could lie as far as 17 billion kilometers from the Earth. [5]

The high temperature of the corona inputs sufficient energy to allow electrons to escape the gravitational pull of the sun. The result of the electron ejections is a charge

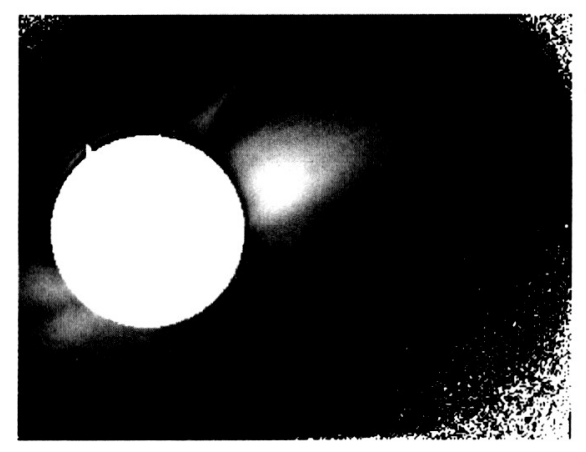

Figure 1: The corona extends several solar diameters. imbalance resulting in the ejection of protons and heavier ions from the corona. The ejected gas is so hot that the particles are homogenized into a dilute plasma. The energy density of the plasma exceeds that of its magnetic field so the solar magnetic field is "frozen" into the plasma.

The electrically neutral plasma streams radially outward from the sun at a velocity of approximately 300 to 900 kilometers per second with a temperature on the order of 104 to $106 \mathrm{~K}$. While the solar wind is millions of metric tons of matter moving at a million kilometers per hour, its density is so low that the physics is that of a vacuum. [5] The energies of the particles range from approximately 0.5 to $2.0 \mathrm{keV} / \mathrm{n}$. The average density of the solar wind is 1 to 30 particles $/ \mathrm{cm}^{3}$. Figure 2 shows that the solar wind velocity and density can vary greatly over a short time period. Table 2 gives the approximate particle composition of the solar wind.

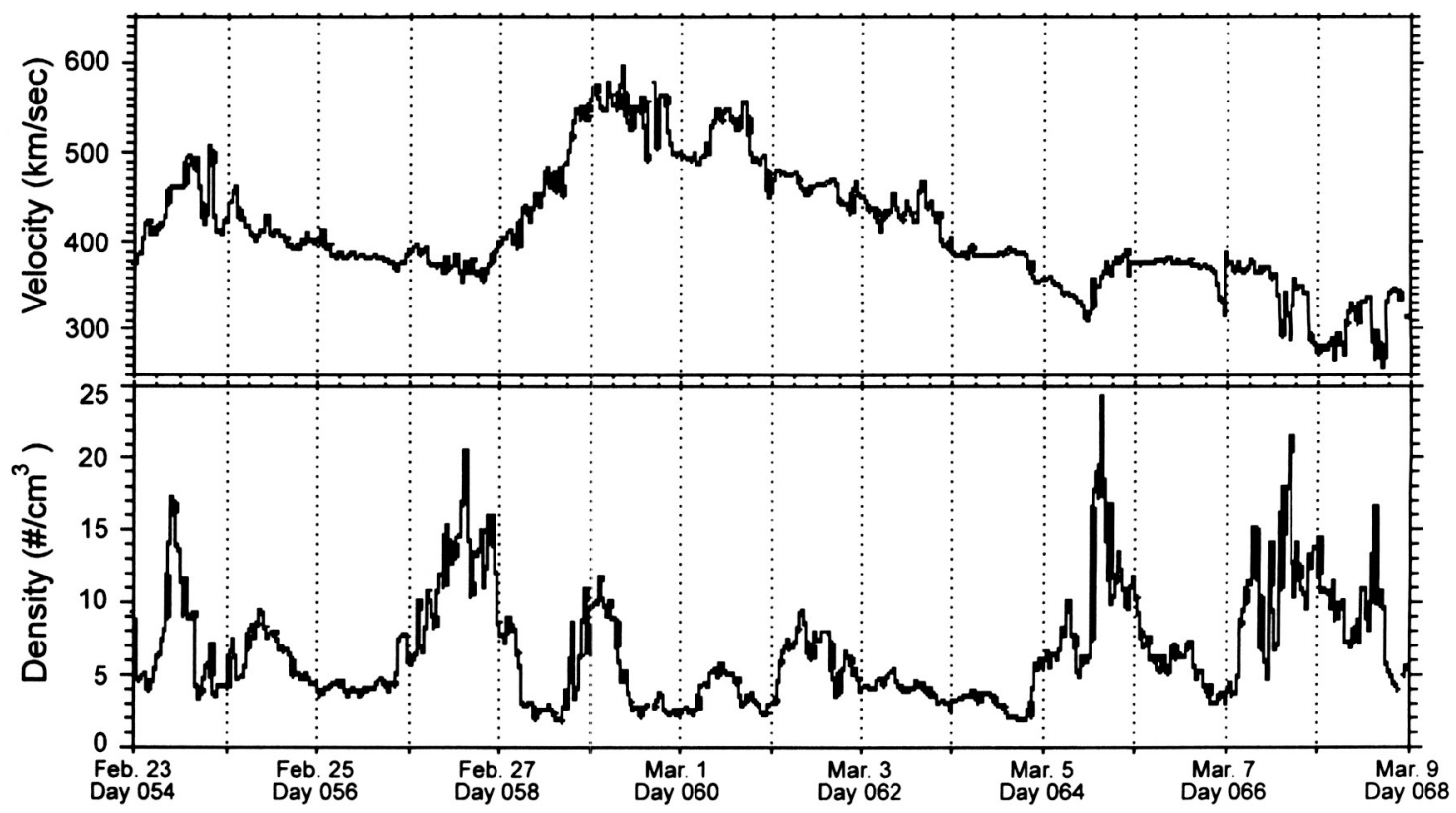

Figure 2: The solar wind velocity and density are highly variable and are a function of the activity on the sun. SOHO/University of Maryland 
Table 2: Solar wind particle composition

\begin{tabular}{||c|c||}
\hline PARTICLE & ABUNDANCE \\
\hline Proton & $95 \%$ of the positively charged particles \\
\hline $\mathrm{He}^{++}$ & $\sim 4 \%$ of the positively charged particles \\
\hline Other Heavy Ions & $<1 \%$ of the positively charged particles \\
\hline Electrons & Number needed to make solar wind neutral \\
\hline
\end{tabular}

It is well known that the level of activity of the sun varies with time defining "solar cycles". The solar cycle as a recurrent pattern of solar magnetic activity was first identified in 1843 by the German observer, Schwabe, who found an approximately 11-year cycle in the number of sunspots* (see Figure 3). The 11-year cycle of sunspots corresponds to similar 11-year cycles of other features in the sun's active regions, including the number of faculae, the rate of incidence of solar flares and coronal mass ejections (CMEs), and the intensity of coronal x-ray and radiofrequency emissions. From Figure 4, it can be seen that the length of the solar cycle can be highly variable. From 1645 to 1715 , the sunspot activity seemed to disappear. Because temperatures on Earth dropped during that time, those 70 years are known as the little ice age. From 1100-1387, there was an increase in the number of sunspots. Studies of recent solar cycles [6,7], Cycles 19 through 22, have determined that the length of the solar cycle over the past 40 years has ranged from 9 to 13 years, with 11.5 being the average. For modeling purposes and for defining the environment for spacecraft missions, the solar cycle can be divided into a 7-year maximum phase of high levels of activity and a relatively "quiet" 4-year minimum

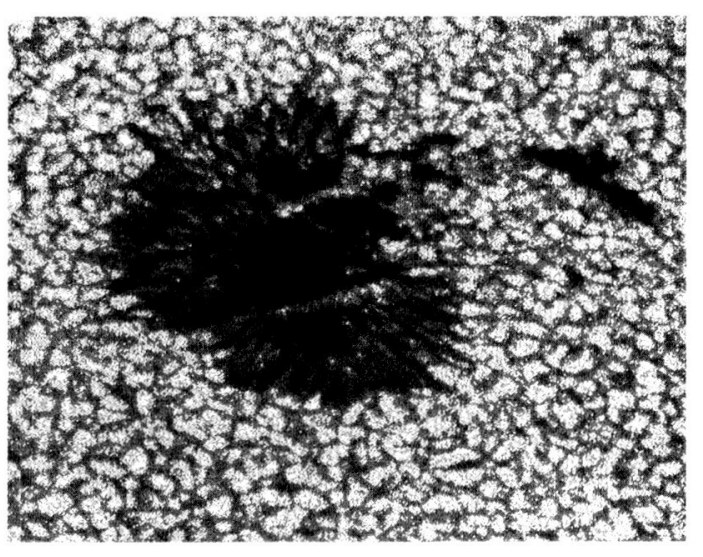

Figure 3: Sunspots are regions of highly dense magnetic field. after Lund Observatory phase.

The space environment is dominated by the activity of the Sun, which acts as both a source and a modulator. It is a source of protons and heavier ions via the periodic high-energy solar events that accelerate large numbers of particles. The solar wind is also a source of the particles trapped in outer regions of the Earth's radiation belts. The galactic cosmic ray heavy ion (GCR) levels follow a cyclic pattern reflecting the activity level of the sun because they originate outside of the solar system and must "fight" against the solar wind to reach interplanetary space. As a result, the GCR levels are highest during solar minimum and lowest during solar maximum. Atmospheric neutrons are secondary products of collisions between GCRs and oxygen or nitrogen atoms in the Earth's atmosphere; therefore, their levels are also modulated by the solar cycle. Finally, the levels of particles trapped in planetary magnetospheres are modulated by both long-term variations in solar activity and solar storm events. The effect of the cyclic variation of the sun's activity will be discussed in more detail in later sections as it applies to specific environments.

\footnotetext{
- cooler areas of the sun seen as dark "spots" through a telescope
} 


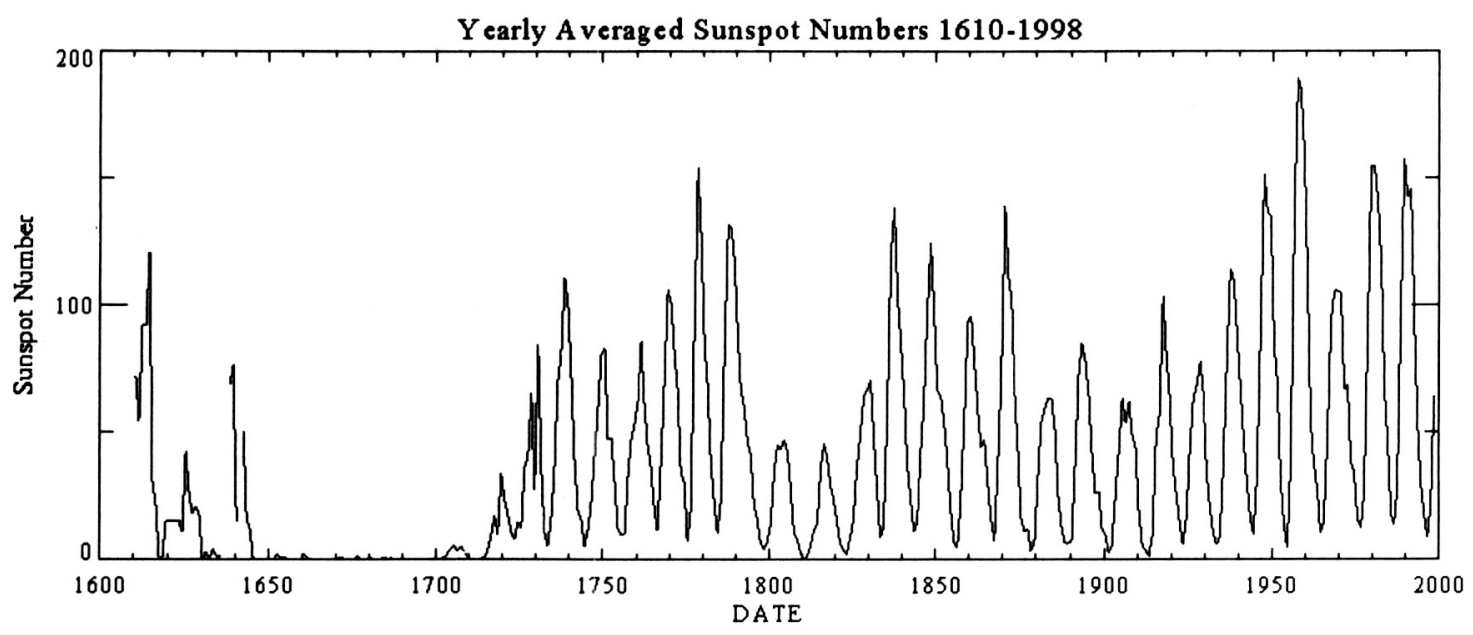

Figure 4: Yearly sunspot numbers

Solar flares and coronal mass ejections (CMEs) are two storm phenomena occurring on the Sun that affect particle levels. Solar flares are seen as sudden brightenings in the photosphere near sunspots (see Figure 5). Flares are intense releases of energy involving tearing and reconnection of strong magnetic field lines. They are the solar systems largest explosive events. Large increases in the solar wind density are measured in interplanetary space after solar flare occurrence because the energy released from the flare accelerates particles in the solar plasma to high energies.

CMEs occur in the chromosphere, the layer of the sun outside of the photosphere. The chromosphere can be seen only when filtering out the bright light of the photosphere. In Figure 6, the chromosphere is seen as a bright rim around the sun. CMEs are observed as large bubbles of gas and magnetic field (see Figure 7). A CME can release approximately $10^{17}$ grams of plasma into interplanetary space. The mechanism for the plasma release is not completely understood. CMEs result in large increases in solar wind velocity. It is the shock wave of the plasma release that is associated with particle acceleration and magnetic storms at the Earth. CMEs are poorly associated with flares but, in very large event CMEs, both CMEs and flares occur together. [8] The particle composition of CMEs and solar flares is discussed in Section 2.9.

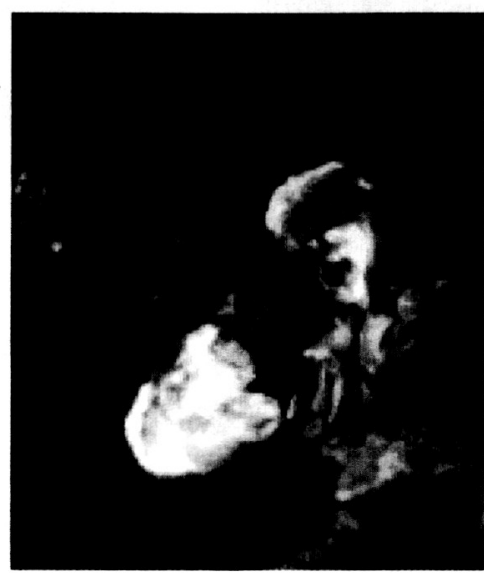

Figure 5: Brightening seen with a solar flare.

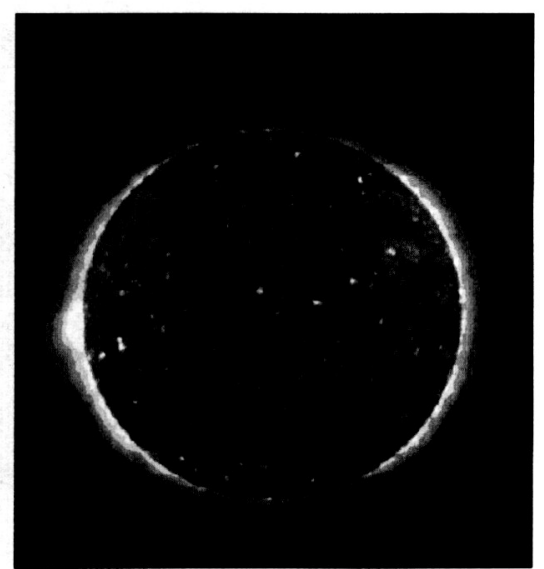

Figure 6: Bright rim around the sun is the chromosphere. 


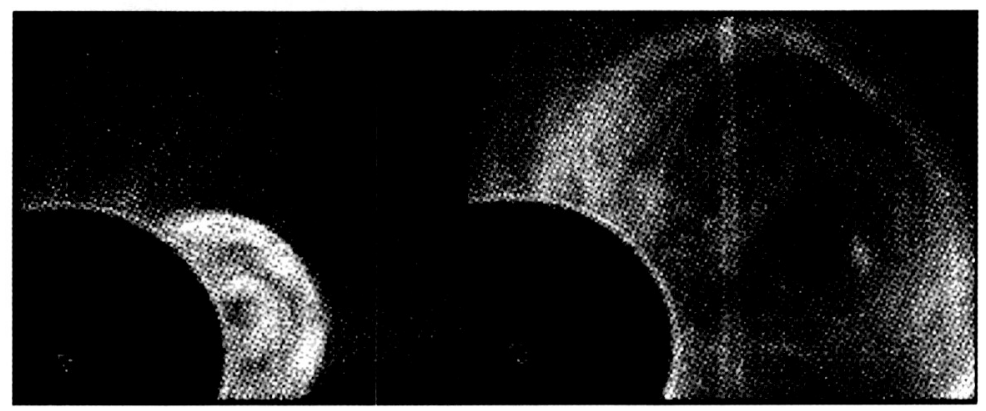

Figure 7: Bubble of gas associated with a coronal mass ejection. NASA/SMM 24 Oct. 1989

\subsection{Meteoroids and Orbital Debris}

Meteoroids are primarily remnants of comet orbits. Several times a year Earth encounters increased meteoroid exposure as it intersects a comet orbit. Also, sporadic particles are released on a daily basis from the asteroid belt. Orbital debris consists of operational payloads, spent rockets stages, fragments of rockets and satellites, and other hardware and ejecta. The United States Air Force Space Command's North American Aerospace Defence Command (NORAD) tracks over 7,000 objects in LEO that are greater than 10 $\mathrm{cm}$ in size, and there are tens of thousands smaller objects. Figure 8 shows the location of the objects tracked by NORAD. From the figure, it is possible to see the large number of objects in the LEO and GEO regions of space where most space agency, military, and commercial operations take place.

Meteoroids and orbital debris are a threat to spacecraft

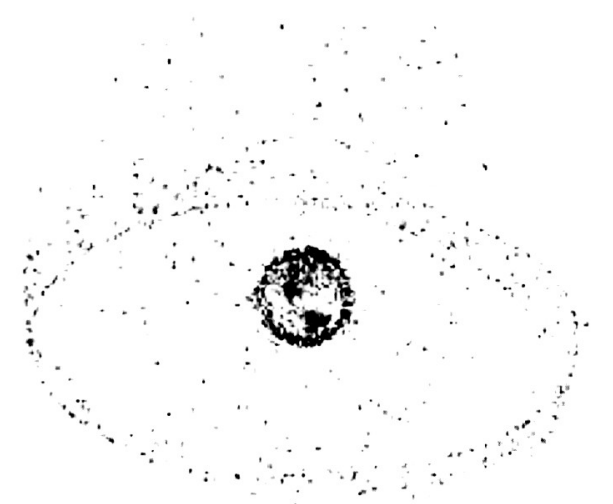

Figure 8: Location of objects tracked by NORAD by causing structural damage and decompression, hypervelocity impacts from larger particles, surface erosion from collisions with smaller objects, and surface effects that cause changes in thermal, electrical, and optical properties. Mission risk factors include increased duration, increased vehicle size, vehicle design, solar cycle, orbit altitude, and inclination, and the threat is highly directional. Koontz et al. [9] give examples of micrometeoroid and orbital debris impacts on the International Space Station (ISS).

\subsection{Ultraviolet Irradiation}

The sun is the natural source of ultraviolet irradiation, which has wavelengths of about 100 to 400 nanometers. UV irradiance can penetrate the atmosphere to reach the surface of the Earth. UV is an important component of the environment to evaluate due to its degradation effects on spacecraft surface materials. It is known to interact with atoms in the atmosphere, particularly oxygen, and ionizing particles to act synergistically on surface materials of spacecraft. UV radiation diffuses with distance from the sun at a rate of $1 / R^{2}$ where $R$ is the radial distance from the Sun. Table 3 lists solar UV irradiance at each planet. 
Table 3: Solar UV irradiance as a function distance from the Sun

\begin{tabular}{||c|c|c||}
\hline \hline PLANET & $\begin{array}{c}\text { DISTANCE } \\
\text { FROM SUN }(\mathrm{AU})\end{array}$ & IRRADIANCE $\left(\mathrm{W} / \mathrm{m}^{2}\right)$ \\
\hline Mercury & 0.39 & $9,126.6$ \\
\hline Venus & 0.72 & $2,613.9$ \\
\hline Earth & 1.00 & $1,367.6$ \\
\hline Mars & 1.52 & 595.0 \\
\hline Jupiter & 5.20 & 51.0 \\
\hline Saturn & 9.54 & 15.0 \\
\hline Uranus & 19.19 & 3.7 \\
\hline Neptune & 30.06 & 1.5 \\
\hline Pluto & 39.53 & 0.9 \\
\hline
\end{tabular}

\subsection{Plasma Environments}

Plasma is ionized gas in which electron and ion densities are approximately equal. Plasma is distinguished from the energetic particle population in that it does not cause radiation effects and has energies $<100 \mathrm{keV}$. Plasma sources are the ionosphere, geomagnetic substorm activity, and the solar wind. The solar wind plasma from the solar corona was discussed in Section 2.1. The ionosphere is the electrically charged portion of the atmosphere and is characterized by low energy (eV) and high density. Plasma from geomagnetic substorm activity, on the other hand, has high energy (keV) and low density. Figure 9 shows the plasma around the Earth as seen by the Extreme Ultraviolet (EUV) instrument on NASA's IMAGE (Imager for Magnetopause-to-Auroral Global Exploration) spacecraft. Plasma shows dramatic variation with altitude, latitude, magnetic field strength, and solar activity. The solar wind plasma was discussed in Section 2.1. The other plasma environments will be discussed in
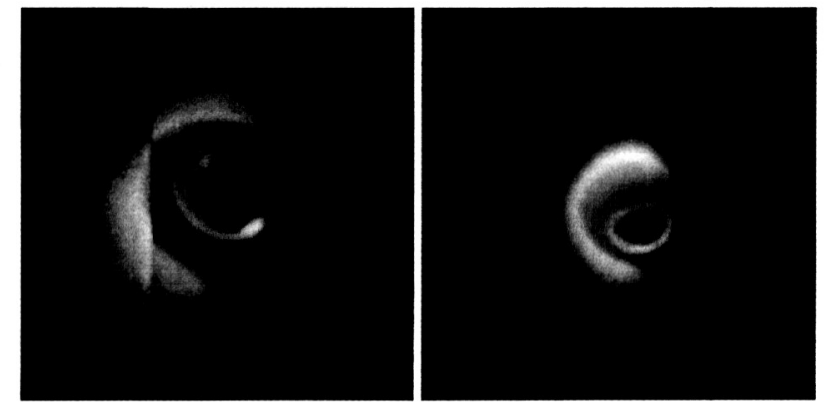

Figure 9. The Helium ion plasma around the Earth as seen by the EUV instrument on the IMAGE spacecraft. Note the auroral activity.

Sections 2.5.2 and 2.7.1.

\subsection{The Earth's Atmosphere}

The Earth's atmosphere is composed of complex layers of matter that are loosely defined by their dominant constituents. Starting from the surface of the Earth, the layers are the troposphere, stratosphere, mesosphere, the neutral thermosphere, and the charged thermosphere (ionosphere). The layers overlap and form a connected system. Figure 10 shows the altitude domains of the regions of the atmosphere. Low altitude spacecraft $(<800 \mathrm{~km})$ are exposed to the environments of thermosphere, so those environments will be discussed in more detail.

\subsubsection{Neutral Thermosphere}

The neutral thermosphere is the neutral portion of the Earth's atmosphere at 90 to $600 \mathrm{~km}$ altitude above the surface of the Earth, composed primarily of neutral gases. In the lower thermosphere, the neutral population is dominated by atomic oxygen and by hydrogen and helium 
in the higher thermosphere. The distribution of the thermosphere neutral gases varies with solar activity because of heating caused by absorption of solar extreme ultraviolet radiation (EUV). A proxy commonly used for EUV is the $10.7-\mathrm{cm}$ radio flux (F10.7).

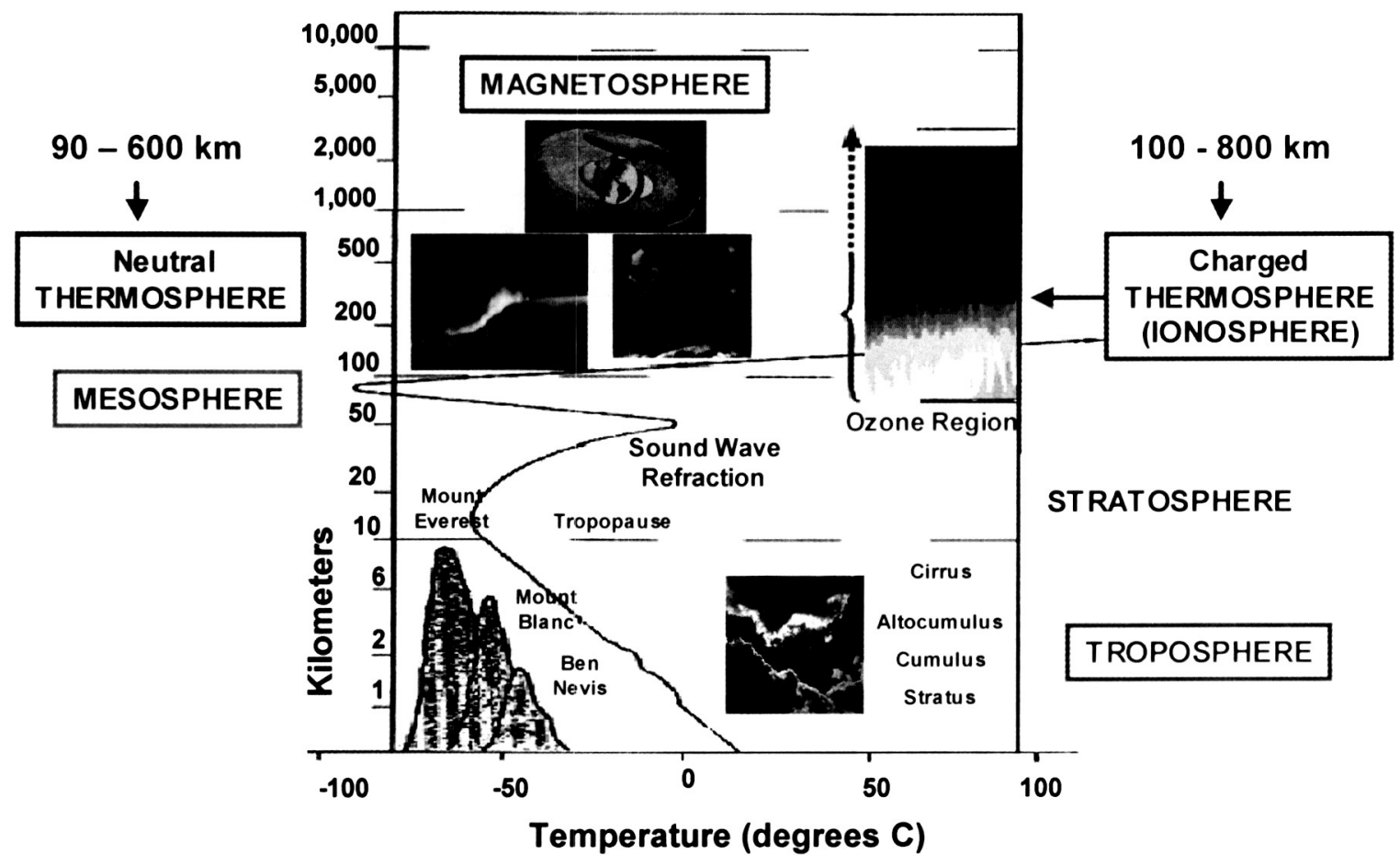

Figure 10: The altitude domains of the Earth's atmosphere, after NASA/MSFC

The main effects of the neutrals on spacecraft are drag, degradation of surface materials, and spacecraft glow. Drag results in altitude decay and torques. Drag is a function of the density of the neutral gas, hence is strongly affected by solar activity. The impact of solar storms on the Earth's atmospheric density often causes sudden changes in the location of tracked objects. Figure 11 is a plot of the number of objects that were lost after a large magnetic storm in March of 1989.

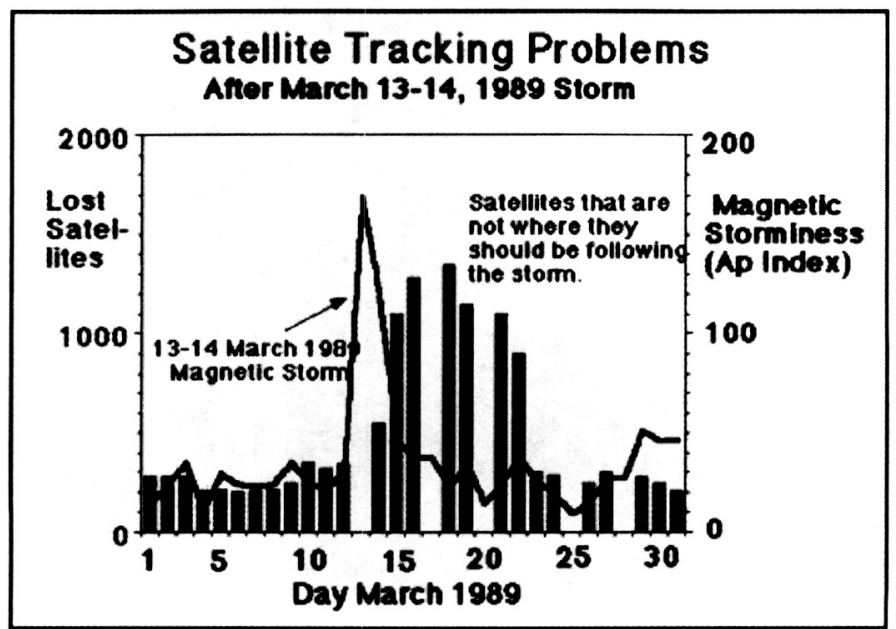

Figure 11: A plot of the number of tracked objects lost after a large magnetic storm 
The degradation of surface materials is also a serious problem in very low earth orbits due to the presence high levels of atomic oxygen at 200 to $400 \mathrm{~km}$. As with other thermosphere constituents, the level of atomic oxygen varies with the solar cycle. The erosion of surface materials causes changes in thermal, mechanical, and optical properties. Micrometeoroid impacts, sputtering, UV exposure, contamination, and ionizing radiation can aggravate these effects. Optical emissions generated by excitation of metastable molecules can also cause spacecraft glow. The surface acts as catalyst, therefore, the effect is material dependent.

\subsubsection{Charged Thermosphere (Ionosphere)}

The Earth's ionosphere is the electrically charged portion of the upper atmosphere from 100 to $800 \mathrm{~km}$ altitude (see Figure 10). It is a low energy (eV) plasma with high density relative to the magnetospheric plasma and the solar wind. Supersonic spacecraft motion through background ions in the ionospheric plasma has detrimental effects on spacecraft in LEO orbits, including solar array coupling to the plasma causing current drain on solar arrays, generation and emission of plasma waves, and increased surface contamination. There is renewed interest in studying the ionosphere because military and civilian communications are severely degraded during storms in the ionosphere induced by solar activity. The disruptions of communications during storms are far reaching as they affect high frequency radio, backscatter radar, satellite communications, and global positioning system (GPS) location.

\subsection{Atmospheres of Other Planets}

Other planets in our solar system have atmospheres which differ dramatically from the Earth's atmosphere. Therefore, designers of missions to other planets must take into account the differences from the Earth's atmosphere and those of other planets and adjust the evaluation and mitigation of the effects accordingly.

The ability of a planet to have an atmosphere is dependent on the planetary surface pressure and gravity (Table 4). For the four "terrestrial planets" the table shows that Mercury and Mars have very thin atmospheres and that the atmosphere of Venus is 92 times that of the Earth. The Jovian planets have large variations in their atmospheric density as a function of radial distance from the center of the planet because they are primarily composed of gases and clouds.

Table 4: Planetary pressure and surface gravity

\begin{tabular}{||c|c|c||}
\hline PLANET & $\begin{array}{c}\text { SURFACE PRESSURE } \\
(\mathrm{BARS})\end{array}$ & $\begin{array}{c}\text { SURFACE GRAVITY } \\
(\mathrm{M} / \mathrm{S})\end{array}$ \\
\hline Mercury & $1.00 \times 10^{-15}$ & 3.70 \\
\hline Venus & 92 & 8.87 \\
\hline Earth & 1 & 9.78 \\
\hline Mars & $8.00 \times 10^{-3}$ & 3.69 \\
\hline Jupiter & $>>100$ & 23.12 \\
\hline Saturn & $>>100$ & 8.96 \\
\hline Uranus & $>>100$ & 8.69 \\
\hline Neptune & $>>100$ & 11.00 \\
\hline Pluto & $3.00 \times 10^{-6}$ & 0.66 \\
\hline
\end{tabular}

In addition to the density of the atmosphere, it is critical to understand the composition of the atmospheres which is shown in Table 5. Notable is the similarity of the great hydrogen planets, Jupiter, Saturn, Uranus, and Neptune and the carbon dioxide atmospheres of Venus and Mars. 
Table 5: Composition of the atmospheres of each planet

\begin{tabular}{|c|c|}
\hline PLANET & ATMOSPHERIC COMPOSITION \\
\hline Mercury & $\begin{array}{c}42 \% \mathrm{O}_{2} \\
29 \% \mathrm{Na} \\
22 \% \mathrm{H}_{2} \\
6 \% \mathrm{He}\end{array}$ \\
\hline Venus & $\begin{array}{c}96 \% \mathrm{CO}_{2} \\
3 \% \mathrm{~N}_{2}\end{array}$ \\
\hline Earth & $\begin{array}{c}78 \% \mathrm{~N}_{2} \\
21 \% \mathrm{O}_{2} \\
1 \% \mathrm{Ar}\end{array}$ \\
\hline Mars & $\begin{array}{c}95 \% \mathrm{CO}_{2} \\
3 \% \mathrm{~N}_{2} \\
2 \% \mathrm{Ar}\end{array}$ \\
\hline Jupiter & $\begin{array}{l}90 \% \mathrm{H}_{2} \\
10 \% \mathrm{He}\end{array}$ \\
\hline Saturn & $\begin{array}{c}96 \% \mathrm{H}_{2} \\
3 \% \mathrm{He}\end{array}$ \\
\hline Uranus & $\begin{array}{l}83 \% \mathrm{H}_{2} \\
15 \% \mathrm{He} \\
2 \% \mathrm{CH}_{4}\end{array}$ \\
\hline Neptune & $\begin{array}{l}80 \% \mathrm{H}_{2} \\
19 \% \mathrm{He} \\
1 \% \mathrm{CH}_{4}\end{array}$ \\
\hline Pluto & $\begin{array}{c}? \\
\mathrm{CH}_{4} ? \\
\mathrm{~N}_{2} \text { Ice? }\end{array}$ \\
\hline
\end{tabular}

\subsection{The Earth's Magnetosphere}

The Earth's magnetosphere is a cavity formed by the interaction of the Earth's magnetic field and the solar wind. In the absence of the solar wind, the Earth's magnetic field would be shaped like the field of a bar magnet; non-varying, nearly symmetric about the magnetic axis, extending outward to long distances, and open at the poles. The bar magnet representation is accurate up to altitude of 4 to 5 Earth radii. The solar wind plasma, with its embedded solar magnetic field, compresses the geomagnetic field until there is balance between the magnetic pressure from the Earth and the momentum pressure from the solar wind forming a "bow shock". On the dayside, during moderate solar wind conditions, the magnetosphere terminates at the magnetopause at $\sim 10$ Earth radii altitude. At the location of this "collisionless" shock, the solar wind plasma cannot penetrate deeply into the geomagnetic field because of its charged particle composition. In fact, $99.9 \%$ of the solar wind particles pass around the Earth's magnetosphere. The flow of the solar wind around the flanks of the magnetopause stretches the geomagnetic field in the anti-solar direction into a long tail of up to $\sim 300$ Earth radii altitude. Some tail field lines are not closed and are connected to the solar magnetic field embedded in the solar wind. Figure 12 shows a depiction of the magnetosphere.

The magnetosphere is filled with plasma that originates from the ionosphere and the solar wind. The plasmasphere is at low and mid latitudes in the inner magnetosphere. The plasma sheet resides in the magnetotail. Overlapping the plasmasphere and the plasma sheet are the highenergy radiation belts or Van Allen belts (named for their discoverer, James Van Allen). Charged particles become trapped because the Earth's magnetic field constrains their motion. They spiral 
around the field lines in a helicoidal path while bouncing back and forth between the magnetic poles. Superimposed on these spiral and bounce motions is a longitudinal drift of the particles because of the gradient of the magnetic field. Figure 13 illustrates the three motions. When the particle makes a complete azimuthal rotation, it has traced a "drift shell". (see Figure 14) The Van Allen belts will be discussed in more detail in Section 2.7.2.

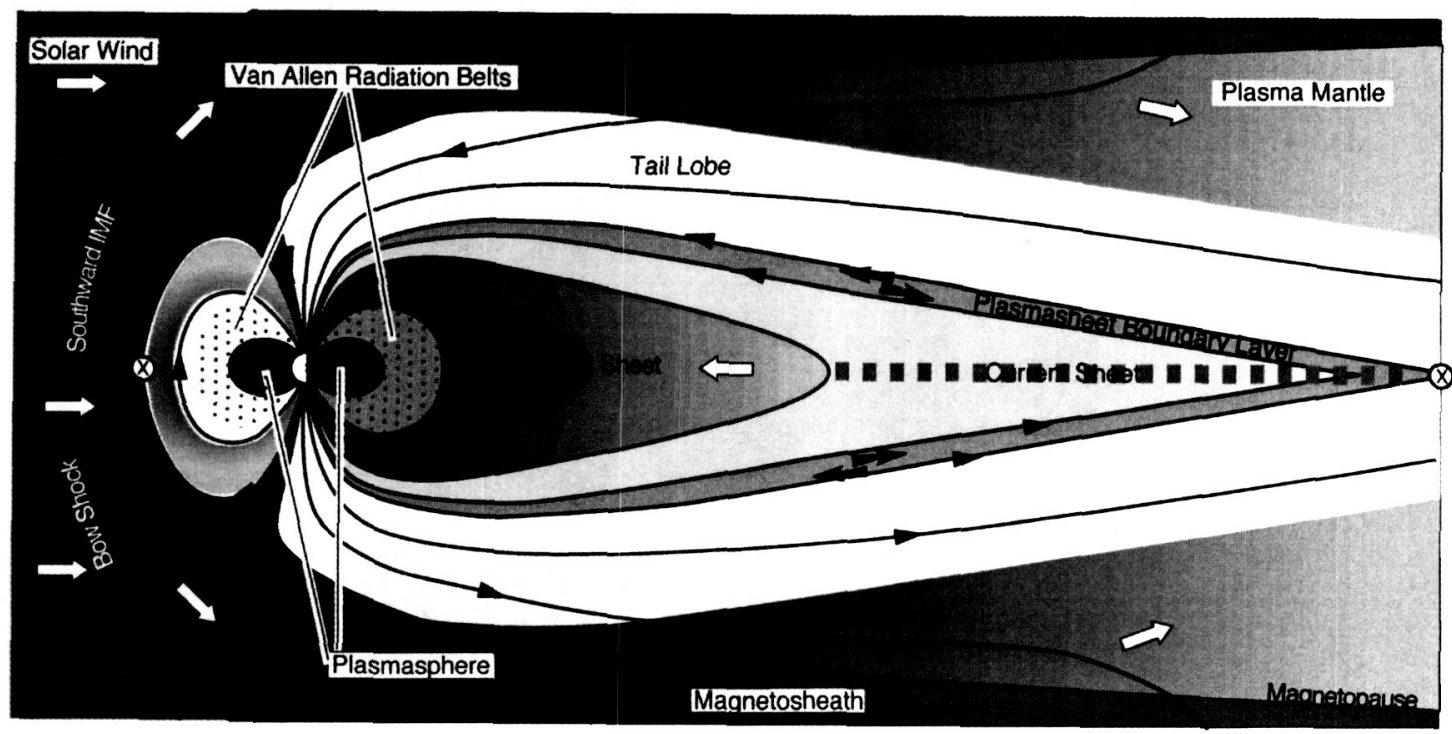

Figure 12: The Earth's magnetosphere, adapted from T. W. Hill by P.H. Reiff

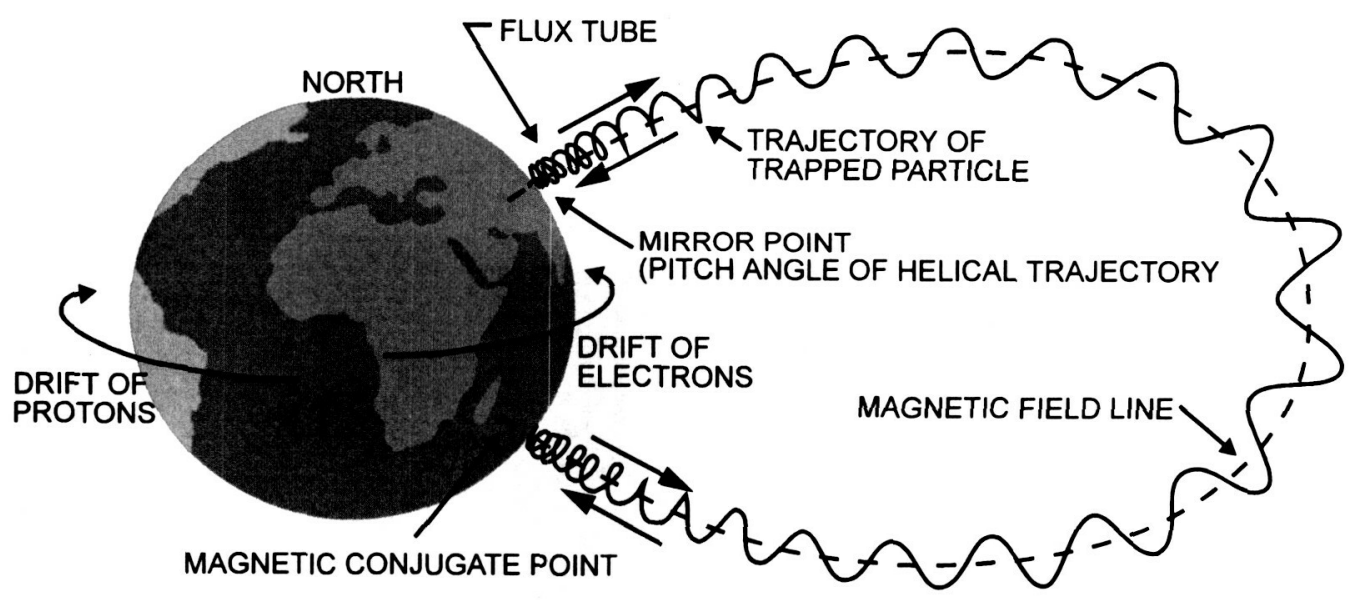

Figure 13: The three motions of the trapped particles form drift shells. after Hess 


\subsubsection{Plasma Storms}

Geomagnetc substorms in the magnetotail plasma sheet can create "hot plasmas" which are injected into near-Earth regions of the magnetosphere. The effects of the plasma injections include biasing of spacecraft instrument readings, acing which causes upsets to electronics, increased current collection, re-attraction of contaminants, and ion sputtering which in turn leads to acceleration of material erosion. Missions affected by these injections are those in GEOs, GTOs, and MEOs. Conditions for the charging effects are large differentials, large fraction of total flux, darkness, and large spacecraft. Satellites at GEO have also measured strong local time effects on the rates of spacecraft charging with most occurring as the satellite passes into the

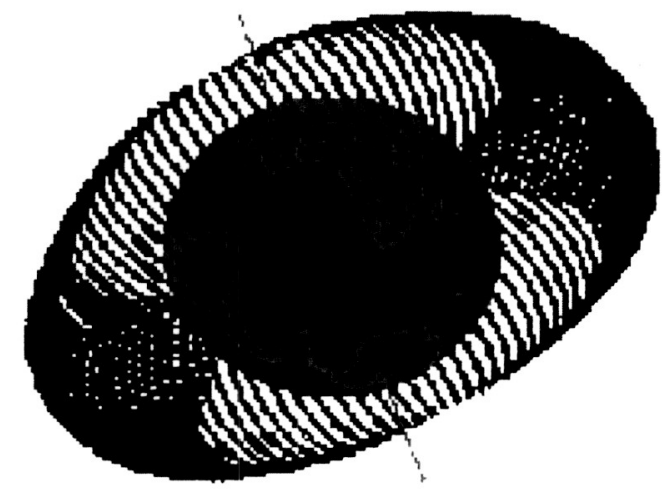

dawn sector.

Figure 14: Drift shell of a trapped particle. Lamarie et al.

\subsubsection{Van Allen Radiation Belts}

The Van Allen belts consist of protons, electrons and heavier ions that are "trapped" on the Earth's magnetic field lines. The trapped electrons have energies up to $10 \mathrm{~s}$ of $\mathrm{MeV}$, and the trapped protons and heavier ions have energies up to $100 \mathrm{~s}$ of $\mathrm{MeV}$. These particles have complex spatial distributions that vary by several orders of magnitude depending on orbit inclination and altitude. The sun is a driver for long and short-term variations in the locations and levels of trapped particles. A feature of the Van Allen belts is the South Atlantic Anomaly (SAA). The $11^{\circ}$ angle between the magnetic and geographic axes and the offset of the geographic and geomagnetic centers of the Earth causes a depression in the magnetic field in the South Atlantic. This magnetic field sink causes charged particles to be trapped at low altitudes $(<1000 \mathrm{~km})$ in that region thereby forming the SAA.

The trapped particles constitute a major radiation hazard for spacecraft. Radiation effects include total ionizing dose (TID), displacements damage, single events effects (SEEs), and deep dielectric charging. In deep dielectric chariging, high-energy trapped electrons can penetrate dielectric materials on a spacecraft and discharge, causing damage to spacecraft circuits and materials. This damage can result in performance degradation or the loss of a mission.

Total ionizing dose is a cumulative effect which causes degradation of microelectronics and materials. As TID accumulates, component performance can be driven outside of their design range. TID is caused by exposure to electrons and protons. Figure 15 is a plot of total ionizing dose in krads of silicon as a function of aluminium shield thickness for various orbits around the Earth. The two curves in the lower half of the graph are for LEOs that pass through the SAA. The 
curves that are higher on the graph are orbits that pass through more intense regions of radiation that are at higher altitudes in the belts. The doses at $>300$ mils of shielding are dominated by the highly energetic trapped protons.

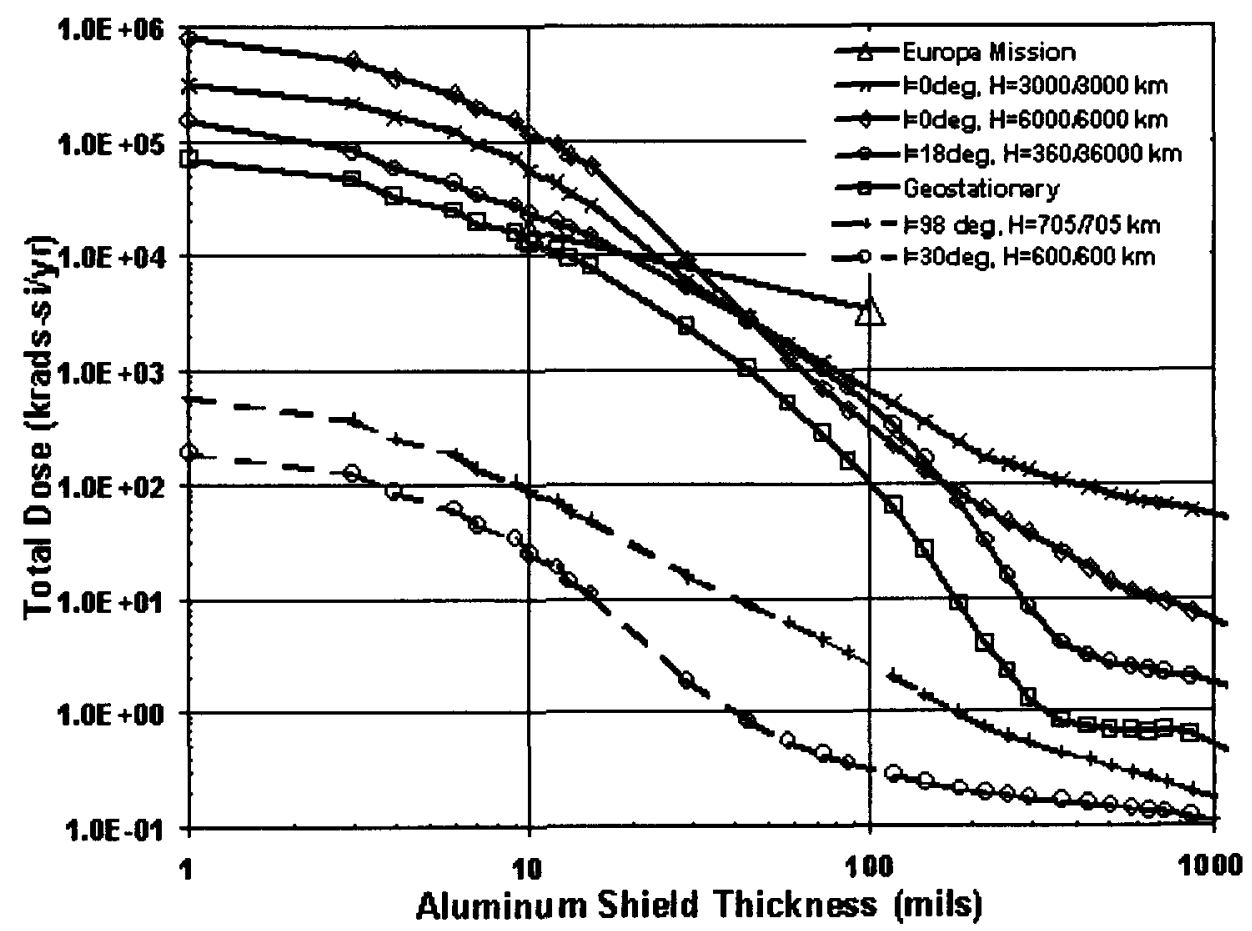

Figure 15: Total ionizing dose-depth curves for various orbits around the Earth

Total non-ionizing dose (also known as displacement damage or bulk damage) is another cumulative effect that causes degradation of solar cells, optocouplers, and focal plane arrays. As particles slow down in material and come to rest they knock atoms out of their lattice location creating defects which increase the resistance of the device. Electrons, protons, and neutrons cause displacement damage, and the energy spectra of the particles are used to evaluate the level of the hazard.

Modern microelectronic systems are plagued by the effects of single particle strikes, namely SEEs, on sensitive regions of devices. There are several types of SEEs, including single event upsets, single event latchups, and single event transients. The consequences of SEEs in systems range from loss of data to the loss of a mission. SEEs are caused by ions from GCRs, solar particle events, and trapped protons. Figure 16 shows the geographic location of single event upsets on the SEASTAR satellite ( $98^{\circ}$ inclination, $705 \mathrm{~km}$ altitude) flight data recorder on a world map of latitude versus longitude. Trapped protons in the SAA cause the concentration of upsets near South America. In fact, the location of the SAA protons is clearly mapped out by the upsets. The upsets that occur in the polar regions are due to galactic cosmic rays and solar particles which, at high latitudes, have access to low altitudes due to the open magnetic field at the poles. 


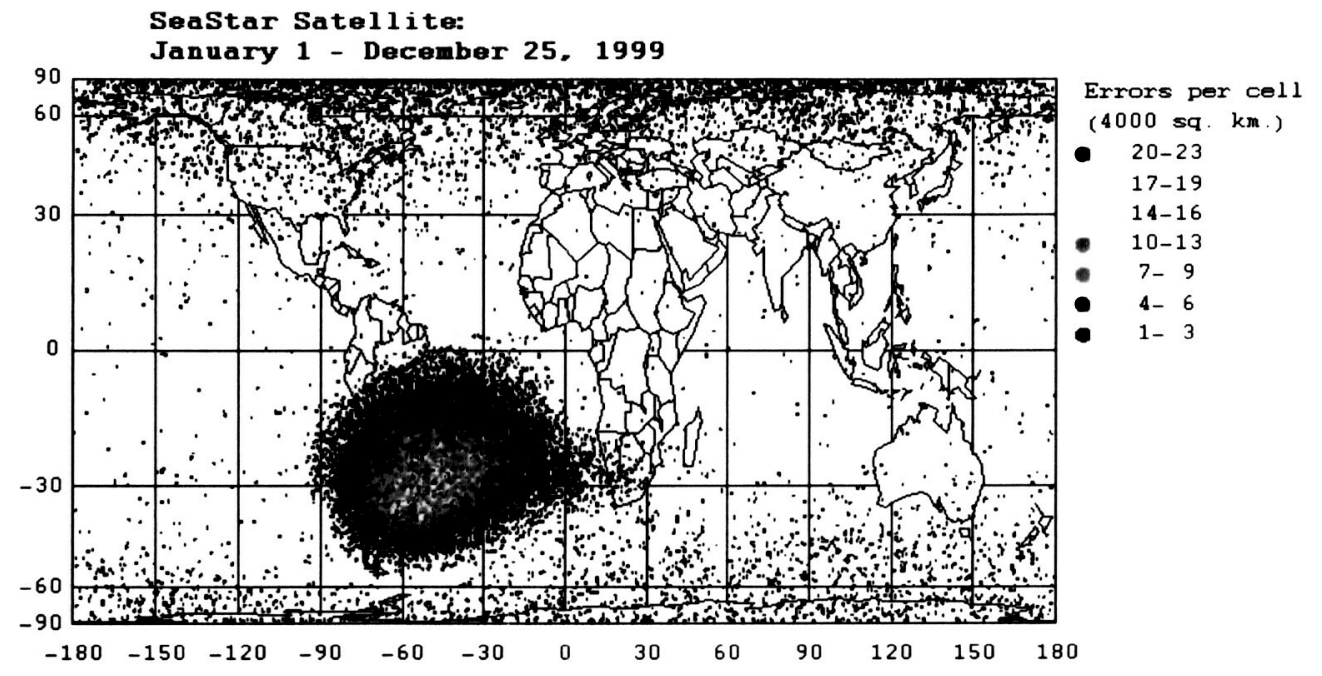

Figure 16: Upsets on the SEASTAR flight data recorder at $705 \mathrm{~km}$ altitude clearly show the location of trapped protons in the South Atlantic Anomaly

\subsection{Magnetospheres of Other Planets}

The minimum requirement for the existence of a planetary radiation belt is that the planet's dipole magnetic moment must be sufficiently great such that the flow of the solar wind is arrested before the particles reach the top of the atmosphere where the particles will lose their energy due to collisions. The magnetic fields of some of the other planets are similar the Earth's, however, they vary in strength. Figure 17 shows a schematic of the relative size of the planetary magnetospheres. Table 6 gives the dipole moments in nanotesta for each of the planets.

Table 6.: Dipole moment for the planets

\begin{tabular}{||c|c||}
\hline PLANET & DIPOLE MOMENT (NT) \\
\hline Mercury & 330 \\
\hline Venus & 0 \\
\hline Earth & 30,760 \\
\hline Mars & 0 \\
\hline Jupiter & 428,000 \\
\hline Saturn & 21,000 \\
\hline Uranus & 22,800 \\
\hline Neptune & 14,200 \\
\hline Pluto & $0 ?$ \\
\hline
\end{tabular}

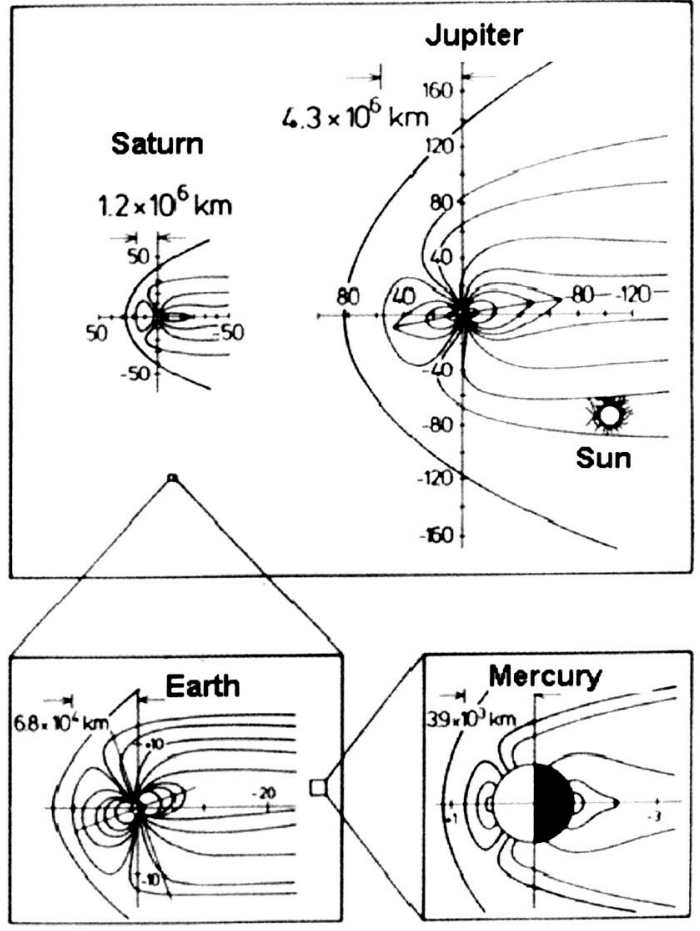

Figure 17: Relative size of planetary magnetospheres 
Table 6 shows that Venus, Mars, and possibly Pluto do not have magnetospheres and, therefore, cannot support particle trapping. Mercury has a weak magnetic field so it is expected it has a trapped particle population proportionally lower than that of the Earth. The Probos probe showed that Mars has a radiation environment, however, it is due to the thin atmosphere of Mars, which allows interplanetary GCRs and solar particles to penetrate to the surface. Interaction of these particles with the atmosphere produces neutrons, which penetrate to the planetary surface and then reflect back. Saturn, Uranus, and Neptune have magnetic fields with similar strength to that of the Earth but measurements indicate that the intensities of the trapped radiation environments of Saturn, and Uranus are much lower than the Earth's and do not pose a threat to spacecraft systems.

Jupiter's enormous magnetic dipole (Table 6) can support an intense particle environment. In fact, its magnetosphere is the largest object in the solar system. Measurements have shown that the radiation environment is considerably more intense than the Earth's and is more extensive, therefore, mission planning for spacecraft that will spend time in trapping regions of Jupiter must include careful definitions of the radiation environment. For example, the electrons at Jupiter have energies of $>100 \mathrm{MeV}$ whereas those at the Earth are in the $10 \mathrm{~s}$ of $\mathrm{MeV}$. Accurate dose calculations require a model that can transport high energy electrons through shielding. Figure 15 shows that the expected dose for a Europa mission is at the megarads level for 100 mils of shielding which is higher than a one year obit around the Earth in the intense MEO regions.

Single event effects are also a problem at Jupiter. In addition to the protons trapped in Jupiter's magnetosphere, single event effects calculations must include oxygen and sulfer ions injected by volcanic activity on lo.

\subsection{Interplanetary Particles}

The sun dominates interplanetary space. Its magnetized plasma, the solar wind, distorts the magnetic field of Earth (see Figure 12) and even the outer planets. In addition to the solar wind plasma, interplanetary space contains high-energy charged particles. This radiation environment consists of galactic cosmic ray particles that are present at all times and particles from solar events that occur sporadically (coronal mass ejections and flares). GCRs cause single event effects on microelectronics on interplanetary missions and on Earth orbiting satellites that spend time over the poles (see Figure 16). Solar protons are both a single event hazard and cause degradation of detectors, microelectronics, and solar cells. The heavy ions from solar particle events can increase the rate of single event effects many factors above the background caused by GCRs.

\subsubsection{Galactic Cosmic Rays}

In the early 1900 s, scientists found that instruments used for studying $x$-rays and radioactivity measured a background source of unidentified radiation. Victor Hess, an Austrian physicist, measured gamma rays by designing ionization chambers and flying them on balloons. With his balloon experiments, he discovered an extremely penetrating radiation that increased in density as altitude increased. From his experiments, he concluded that this radiation was from an extraterrestrial source. Later, Jacob Clay was able to show that cosmic rays were the source of the on-ground radiation and that measured by Hess higher in the atmosphere. In 1936, Hess received the Noble Prize for the discovery of galactic cosmic rays. Although we now know that these "rays' are really particles, they are still referred to as cosmic rays.

The GCRs originate outside of the solar system. Although there are plausible models of how they are produced, their origin is still a matter of debate. [10] Scientists believe that they 
propagate through all space that is unoccupied by dense matter. They are essentially isotropic outside of regions of space that are dominated by particles and fields of the sun. Galactic radiation consists of ions of all elements of the periodic table and is composed of about $83 \%$ protons, $13 \%$ alphas (4He ions), $3 \%$ electrons, and about $1 \%$ heavier nuclei. Unlike the charged particles that originate at the Sun, the GCRs do not have a characteristic energy limit. Their energies range from $10 \mathrm{~s}$ of $\mathrm{MeV} / \mathrm{n}$ to $100 \mathrm{~s}$ of $\mathrm{GeV} / \mathrm{n}$. Because they must pass through about $7 \mathrm{~g} / \mathrm{cm}^{2}$ of interstellar gas, the GCRs of even the heaviest ions are probably fully ionized. [11]

A second source of galactic particles is the so-called "anomalous component". It is composed of helium and heavier ions with energies greater than $50 \mathrm{MeV} /$ nucleon. It is believed that the anomalous component originates in the neutral interstellar gas that diffuses into the heliosphere, becomes singly ionized by solar radiation or charge exchange, and is then connected by the solar wind to the outer heliosphere. The ions are then accelerated and propagate to Earth. The anomalous component is seen only during solar minimum and the details vary from solar minimum to solar minimum. There is growing evidence that the anomalous component is singly ionized, therefore, the ions have greater ability to penetrate the magnetosphere.

Our knowledge of the abundances of galactic cosmic rays comes from spacecraft and balloon experiments that have been conducted over a forty-year period. Figure 18 from Medwaldt [12] gives the abundances of the heavy ions at an energy of $2 \mathrm{GeV} / \mathrm{n}$ as a function of particle nuclear charge $\mathrm{z}$. The values are normalized to silicon $=10^{6}$. Note that the relative flux intensities vary by several orders of magnitude. The relative abundances are roughly proportional to the distribution in solar system material. Significant differences are discussed in Medwaldt [12] who also gives a table of relative abundances.

The galactic particles are always present, however, their intensities rise and fall with the solar cycle variations. The sun modulates a set of local interstellar spectra at the outer boundary of the heliosphere. [13] The modulation can be defined by a single parameter which is a function of distance from the sun, the speed of the radial solar wind, and a radial transport particle diffusion coefficient. GCRs are at their peak level during solar minimum and at their lowest level during solar maximum and we now know that the length of the GCR modulation cycle is 22 years and not 11 years as previously thought. The difference between the extremes of the solar minimum and maximum fluence levels is approximately a factor of 2 to 10 depending on the ion energy. Figure 19 shows the slow, long-term

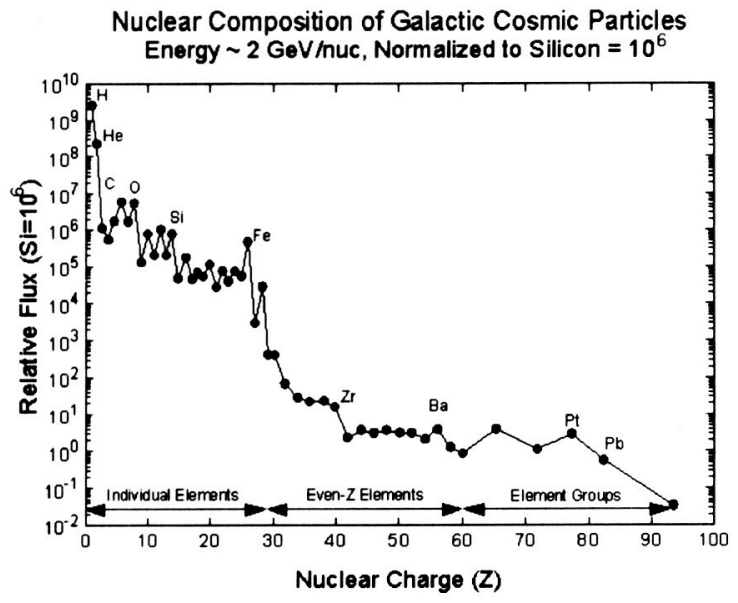

Figure 18: Relative abundances of galactic cosmic ray ions in interplanetary space. after Medwaldt

CNO - 24 Hour Averaged Mean Exposure Flux

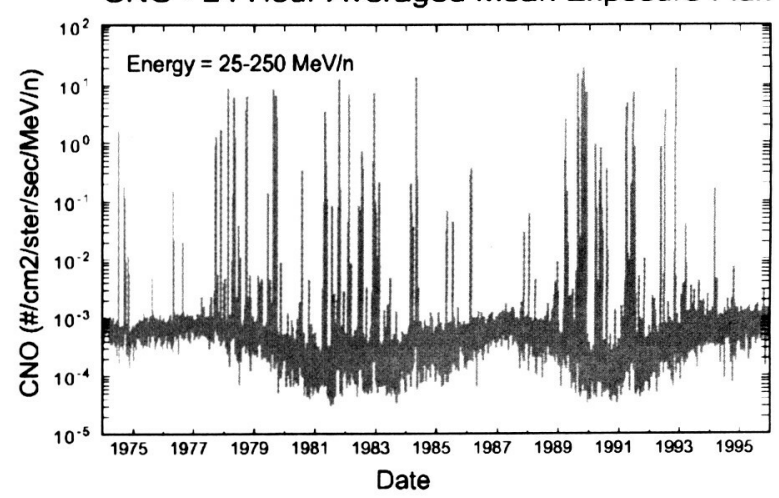

Figure 19: IMP-8 measurements of interplanetary ions from the $\mathrm{C}-\mathrm{N}-\mathrm{O}$ group. Note the solar particle event spikes superimposed on the lower level, slowly varying galactic cosmic rays. after Nakamura 
cyclic variation of the cosmic ray $(\mathrm{C}, \mathrm{N}, \mathrm{O})$ fluences for a 20 -year period as measured by the IMP-8 spacecraft. The sharp spikes superimposed on the cosmic ray background are caused by solar events.

Measurements from Pioneer and Voyager show that the composition of cosmic rays is weakly dependent on the distance from the Sun. The radial gradient from 0.3 to $40 \mathrm{AU}$ is $<10 \%$ per $\mathrm{AU}$. For the anomalous component, the gradient increases to $15 \%$ per AU. During solar maximum there is $0 \%$ gradient out to $30 \mathrm{AU}$. Latitude gradients have also been studied and found to be $0.5 \%$ per degree and 3-6\% per degree for the anomalous component. [14]

The Earth's magnetic field provides some protection from the galactic particles by deflecting the particles as they impinge upon the magnetosphere. The penetration power of these particles is a function of the particle's energy and ionization state. The exposure of a spacecraft primarily depends on the inclination and, secondarily, the altitude of the trajectory. Cosmic rays have free access over the polar regions where field lines are open to interplanetary space. (see Figure 16)

\subsubsection{Solar Particles}

The sun is always active but it has been observed that there is a definite periodicity to the level of activity. Thus, the solar cycle is divided into minimum and maximum phases (see Section 2.1). During the maximum phase of the solar cycle, the numbers and intensity of coronal mass ejections and solar flares increases. This causes periodic increases in the levels of interplanetary particles up to orders of magnitude over the GCR environment. These particles consist of ions of all elements but protons usually dominate the abundances. As with the GCRs, spacecraft receive some protection from solar particles by the Earth's magnetosphere depending on their orbit. Analysis of the exposure of spacecraft orbiting the Earthas a function of the geomagnetic disturbances that are often associated with solar events is especially critical. For example, CRRES data showed that solar protons reached $L$ shell values as low as 2. [15] Also, unlike galactic heavy ions, which are, for the most part, fully ionized, solar heavy ions are more often singly ionized because they pass through less matter before reaching the Earth. This must be taken into account when calculating the degree of penetration of the solar particles into the magnetosphere.

Solar particles diffuse as they move through the interplanetary medium. Therefore, the solar particles abundances are a function of radial distance from the Sun. There are few accurate measurements of solar particles throughout the Solar System so the models of the particle diffusion are not accurate. Table 7 gives estimates of the scaling factors that are commonly used to calculate solar particle levels at distances other than the Earth.

Table 7: Scaling factors for solar particles levels at regions $\neq 1 \mathrm{AU}$

\begin{tabular}{|c|c|}
\hline RADIAL DISTANCE & RADIAL SCALING FACTOR RANGE \\
FROM THE SUN (AU) & \\
\hline$<1 \mathrm{AU}$ & $1 / \mathrm{R}^{2}$ to $1 / \mathrm{R}^{3}$ \\
\hline$>1 \mathrm{AU}$ & $1 / \mathrm{R}$ to $1 / \mathrm{R}^{2}$ \\
\hline
\end{tabular}

\subsubsection{Solar Protons}

Figure 20 shows the particle counts for $\mathrm{E}>10$ and $>30 \mathrm{MeV}$ for some of the larger solar proton events for solar cycles 20,21 , and 22 (measured by the GOES spacecraft). Superimposed on the solar event data is the number of sunspots. Note that although the number of proton events is greatly reduced during solar minimum, they still can and do occur. Also, the figure shows that the peak of proton event activity for each solar cycle usually does not correspond to the peak sunspot number. Solar cycles vary in severity in terms of solar proton events. For example, in cycle 21 there were no proton events as large as the August 1972 event of cycle 20 whereas there 
were at least six events in cycle 22 where the intensity exceeded $10^{9}$ protons $/ \mathrm{cm}^{2}$ for energies greater than $30 \mathrm{MeV}$. The energies of the solar event protons may reach a few hundred $\mathrm{MeV}$. The duration of the events is from several hours to a few days.

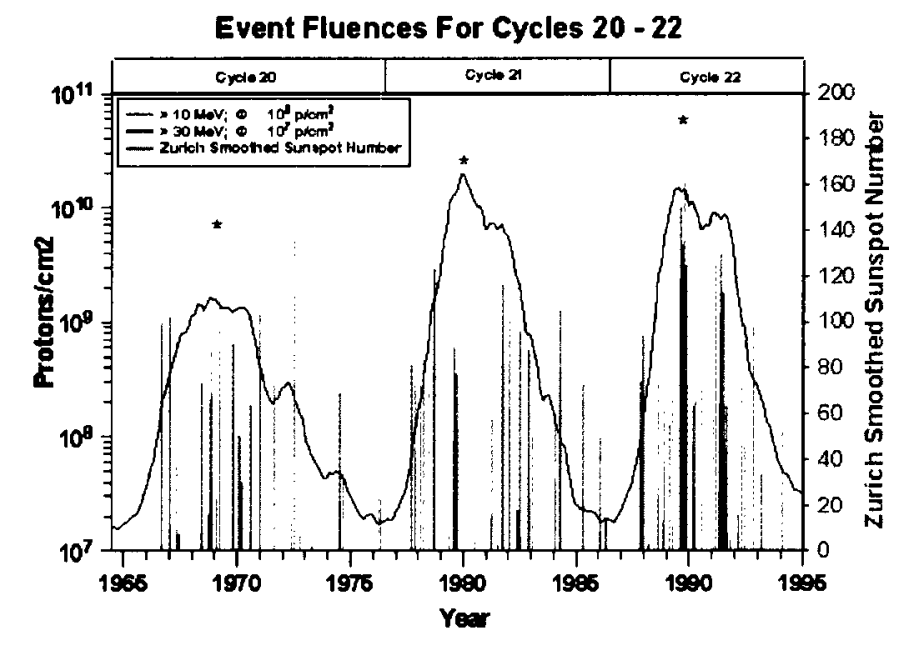

Figure 20: Large solar proton events for solar cycles 20, 21, and 22. The number of sunspots is superimposed on the graph.

\subsubsection{Solar Ions - Helium and Higher}

Some solar particle events are heavy ion rich with energies ranging from $10 \mathrm{~s}$ of $\mathrm{MeV} / \mathrm{n}$ to $100 \mathrm{~s}$ of $\mathrm{GeV} / \mathrm{n}$. For the 26 events observed on CRRES [13], the peak fluxes for the helium ions with energies $\mathrm{E}>40 \mathrm{MeV} / \mathrm{n}$ were three times higher than the galactic cosmic ray heavy ion levels. Above energies of a few hundred $\mathrm{MeV} / \mathrm{n}$ to approximately $1000 \mathrm{MeV} / \mathrm{n}$ (depending on the element), the solar helium levels merge with those of the galactic cosmic ray background. Early attempts to characterize the solar heavy ions at higher energies were restricted by a limited dataset. Later more space data became available. Dietrich et al. [16] used data from the University of Chicago's Cosmic Ray Telescope on the IMP-8 and GOES satellites to study the heavy ion events. They analyzed high energy spectra for $\mathrm{C}, \mathrm{O}$, and $\mathrm{Fe}$ using direct measurements and determined fluences in one or two energy bins for $\mathrm{N}, \mathrm{Ne}, \mathrm{Mg}, \mathrm{Si}, \mathrm{S}, \mathrm{Ar}$, and $\mathrm{Ca}$. Also, $\mathrm{He}$ fluences were studied using carbon indices. This dataset provides the most comprehensive picture of high-energy solar heavy ions to date.

\section{SUMMARY}

There are many unknowns in space environments and the interaction mechanisms. The low level of funding in the applied science discipline of spacecraft environments and effects has resulted in model development and validation lagging behind rapid technology changes. Access to space for validating effects models and ground test protocols is also critically low. Ground tests cannot duplicate the space environment, particularly when environments have synergistic effects and effects are complicated by enhanced low dose rates in space. Often these unknowns require that large design margins be applied to performance predications, resulting in overheads that reduce capability or that can preclude use of newer technologies in spacecraft systems. 


\section{References}

[1] K. A. LaBel, A. H. Johnston, J. L. Barth, R. A. Reed, and C. E. Barnes, "Emerging Radiation Hardness Assurance (RHA) issues: A NASA approach for space flight programs," IEEE Trans. on Nucl. Science, Vol. 45, No. 2, December 1998

[2] J. D. Kinnison, "Radiation effects in the New Millennium - Old Realities and New Issues, Section V: Achieving Reliable, Affordable Systems," 1998 IEEE Nuclear and Space Radiation Effects Conference Short Course, July 20, 1998, Newport Beach, CA.

[3] J. L. Barth, "Applying Computer Simulation Tools to Radiation Effects Problems, Section II: Modeling Space Radiation Environments," 1997 IEEE Nuclear and Space Radiation Effects Conference Short Course, July 21, 1997, Snowmass Village, CO.

[4] A. Holmes-Siedle and L. Adams, Handbook of Radiation Effects Second Edition, Oxford Press, 2002.

[5] J. M. Nash, "Cosmic Storms Coming,", TIME, pp. 54-55, September 9, 1996.

[6] E. G. Stassinopoulos, G. J. Brucker, D. W. Nakamura, C. A. Stauffer, G. B. Gee, and J. L Barth, "Solar Flare Proton Evaluation at Geostationary Orbits for Engineering Applications," IEEE Trans. on Nucl. Science, Vol. 43, No. 2, pp. 369-382, April 1996.

[7] J. Feynman, T. P. Armstrong. L. Dao-Gibner, and S. Silverman, "New Interplanetary Proton Fluence Model," J. Spacecraft, Vol. 27, No. 24, pp 403-410, July-August 1990.

[8] D. V. Reames, "Solar Energetic Particles: A Paradigm Shift," Revs. Geophys. (Suppl.), 33, 585, 1995.

[9] S. L. Koontz, M. Pedley, R. R. Mikatarian, J. Golden, P. Boeder, J. Kern, H. Barsamian, J. I. Minow, R. L. Altstatt, M. J. Lorenz, B. Mayeaux, J. Alred, C. Soares, E. Christiansen, T. Schneider, and D. Edwards," Materials Interactions with the Space Environment: International Space Station - May 2000 to May 2002," these proceedings.

[10] J. W. Cronin, T. K. Gaisser, and S. P. Swordy, "Cosmic Rays at the Energy Frontier," Scientific American, January 1997.

[11] J. H. Adams, Jr., R. Silberberg, and C. H. Tsao, "Cosmic Ray Effects of Microelectronics, Part I: The Near-Earth Particle Environment," NRL Memorandum Report 4506, August 25, 1981.

[12] R. A. Medwadlt, "Elemental Composition and Energy Spectra of Galactic Cosmic Rays," Proc. from Conference on Interplanetary Particle Environment, JPL Publication 88-28, pp. 121-132, JPL, Pasadena, CA, April 15, 1988.

[13] D. L. Chenette, J. Chen, E. Clayton, T. G. Guzik, J. P. Wefel, M. Garcia-Munoz, C. Lapote, K. R. Pyle, K. P. Ray, E. G. Mullen, and D. A. Hardy, "The CRRES/SPACERAD Heavy Ion Model of the Environment (CHIME) for Cosmic Ray and Solar Particle Effects on Electronic and Biological Systems in Space," IEEE Trans. on Nucl. Science, Vol. 41, No. 6, pp. 2332-2339 December 1994.

[14] R. B. McKibben, "Gradients of Galactic Cosmic Rays and Anomalous Components," Proc. from Conference on Interplanetary Particle Environment, JPL Publication 88-28, pp. 135-148, April 15, 1988.

[15] M. S. Gussenhoven, E. G. Mullen, and D. H. Brautigam, "Improved Understanding of the Earth's Radiation Belts from the CRRES Satellite," IEEE Trans. on Nucl. Science, Vol. 43, No. 2, April 1996.

[16] W. F. Dietrich, A. J. Tylka, and P. R. Boberg, "Probability Distributions of High-Energy Solar-Heavy-Ion Fluxes from IMP-8: 1973-1996," to be presented at IEEE/NSREC Conference 1997. 